\title{
Analytic solutions for reduced leading-edge noise aerofoils
}

\author{
Lorna J. Ayton * and Chaitanya Paruchuri ${ }^{\dagger}$
}

\begin{abstract}
This paper presents an analytic solution for the sound generated by an unsteady gust interacting with a semi-infinite flat plate with a piecewise linear periodic leading edge. The Wiener-Hopf method is used in conjunction with a non-orthogonal coordinate transformation and separation of variables to allow analytical progress. A fully analytic solution is obtained in terms of a modal expansion for the far-field noise which is obtained by summing only a finite number of cuton modes, allowing very quick evaluation. The analytic solution is compared to experimental results for five test case leading-edge geometries. Good agreement is seen indicating the analytic model is capturing the key features of the interaction such as the destructive interference from the tip and root. In four of the five test cases the serrated edges show large reductions of noise compared to the straight edge at mid and high frequencies, however the square wave geometry is seen to be ineffective at noise reduction for high frequencies.
\end{abstract}

\section{Introduction}

Leading-edge noise is a common and unavoidable source of noise generated by aeroengines when the wakes from forward rotor rows interact with rearward stator rows. Noise generated in this way can be reduced by altering the geometry of the blades, typically blade thickness, camber and angle of attack $)^{1-3}$ however within the confines of an aeroengine this adaptation is not always practical. Therefore it is important to develop different alterations to the blades which will reduce noise but that are appropriate for use within an aeroengine.

A popular adaptation to reduce leading-edge noise is to alter the spanwise straight leading edge of a blade to be serrated, which mimics the leading edges of owls' wings. ${ }^{4,5}$ A single-frequency serration is the most common adaptation investigated numerically,${ }^{6-8}$ experimentally, ${ }^{9-12}$ and analytically. ${ }^{13-16}$ Whilst these analytical solutions use very simplified models of convective gusts scattering off of semi-infinite flat plates with leading-edge serrations, they can highlight key noise-reduction mechanisms that can only be speculated on from experimental and numerical results.

The single-frequency serration is believed to be effective in reducing leading-edge noise due to a destructive interference of scattered acoustics in the far field, and a redistribution of acoustic energy from cuton modes to cutoff modes with increasing serration tip-to-root heights. ${ }^{13}$ With this understood the task is now to determine if different leading-edge geometries will result in better noise reductions through either an increased destructive interference, or greater ability to redistribute energy to the cutoff modes.

This paper therefore presents an analytic solution for the noise generated by a convective gust interacting with a semi-infinite flat plate with an arbitrary piecewise linear leading edge. To do so, we generalise the method used in Ayton \& $\mathrm{Kim}^{13}$ which considered specifically the case of a single-frequency serration, to a more general leading-edge geometry. We compare the analytic predictions against experimental results.

\section{Formulation of the problem}

We consider the interaction of a convective unsteady gust in uniform flow of Mach number $M$ over a semiinfinite flat plate with a spanwise periodic leading edge. We non-dimensionalise lengths by the wavelength of the periodic leading-edge geometry, $\lambda^{*}$, and velocities by the upstream mean flow velocity, $U^{*}$. We consider the spanwise region $0 \leq z \leq 1$, and impose periodic boundary conditions across $z=0,1$. The blade lies in

*EPSRC Early Career Fellow, Churchill College and DAMTP, University of Cambridge, L.J.Ayton@damtp.cam.ac.uk

${ }^{\dagger}$ Research Scholar, Faculty of Engineering and the Environment, University of Southampton 
region $x>\tilde{c} F(z), y=0$, with $x$ denoting the streamwise direction, and $\tilde{c}$ permitting a variable 'tip-to-root' ratio, i.e. a varying difference of the height of the leading-edge geometry.

We restrict the leading-edge geometry such that $F(z)$ is a piecewise linear function, therefore we can investigate most commonly used geometries such as the serration, triangular wave, and slitted root serration easily. Here we consider the five leading-edge geometries depicted in Figure 1, although the results will be applicable to any piecewise linear geometry (including piecewise linear approximations of any continuous periodic leading edge geometry such as a sinusoid), and dual-frequency serrations.

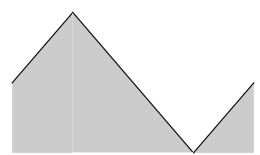

(a) Sawtooth

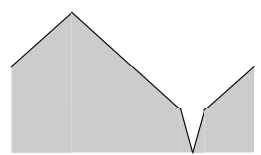

(b) Slitted v-root

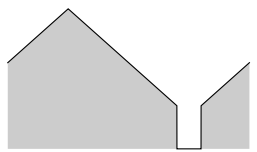

(c) Slitted u-root

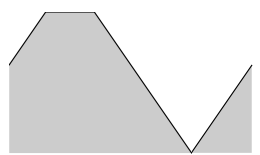

(d) Chopped peak

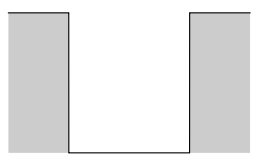

(e) Square wave

Figure 1: Leading-edge geometries over one wavelength.

Note in all geometry cases, the $z=0,1$ boundaries of the periodic function are chosen to be away from any regions of discontinuities of the leading edge. This ensures the solution correctly captures any influence of the discontinuities.

The unsteady gust incident from far upstream takes the form

$$
\mathbf{v}_{g}=\mathbf{A} \mathrm{e}^{\mathrm{i} k_{1} x+\mathrm{i} k_{2} y+\mathrm{i} k_{3} z-\mathrm{i} \omega t},
$$

where the amplitude, $\mathbf{A}=\left(A_{1}, A_{2}, A_{3}\right)^{T}$, is constant. For simplicity we shall take $\mathbf{A}=(0,1,0)$.

As for the specific serration case in Ayton \& $\mathrm{Kim}^{13}$ we decompose the unsteady flow field into a convective gust part and an acoustic response part, $\mathbf{v}=\mathbf{v}_{g}+\mathbf{v}_{a}$, and write the response as $\mathbf{v}_{a}=\nabla G$. We suppose $G$ is harmonic in time $\sim \mathrm{e}^{-\mathrm{i} \omega t}$ therefore spatially satisfies the convected Helmholtz equation,

$$
\beta^{2} \frac{\partial^{2} G}{\partial x^{2}}+\frac{\partial^{2} G}{\partial y^{2}}+\frac{\partial^{2} G}{\partial z^{2}}+2 \mathrm{i} k M \frac{\partial G}{\partial x}+k^{2} G=0,
$$

where $\beta^{2}=1-M^{2}$ and $k=\omega / c_{0}$ with $c_{0}$ the speed of sound of the background steady flow. Since the gust convects with the background flow, we require $k=k_{1} M$. The zero normal velocity boundary condition on the aerofoil surface requires

$$
\left.\frac{\partial G}{\partial y}\right|_{y=0}=-\mathrm{e}^{\mathrm{i} k_{1} x+\mathrm{i} k_{3} z} \quad x>\tilde{c} F(z) .
$$

We also impose continuity of the potential upstream

$$
\left.\Delta G\right|_{y=0}=0 \quad x<\tilde{c} F(z)
$$

and a periodic condition across $z=0,1$,

$$
\left.G\right|_{z=1}=\left.G\right|_{z=0} \mathrm{e}^{\mathrm{i} k_{3}},\left.\quad \frac{\partial G}{\partial z}\right|_{z=1}=\left.\frac{\partial G}{\partial z}\right|_{z=0} \mathrm{e}^{\mathrm{i} k_{3}}
$$

To simplify the governing equation, (2), we apply a convective transform,

$$
h=G(x, y, z) \mathrm{e}^{\mathrm{i} k_{1} M^{2} x / \beta^{2}},
$$

to eliminate the convective terms. The resulting governing equation and boundary conditions for $h(x, y, z)$ are

$$
\begin{gathered}
\beta^{2} \frac{\partial^{2} h}{\partial x^{2}}+\frac{\partial^{2} h}{\partial y^{2}}+\frac{\partial^{2} h}{\partial z^{2}}+\left(\frac{k_{1} M}{\beta}\right)^{2} h=0, \\
\left.\frac{\partial h}{\partial y}\right|_{y=0}=-\mathrm{e}^{\mathrm{i} \frac{k_{1}}{\beta^{2}} x+\mathrm{i} k_{3} z} \quad x>\tilde{c} F(z), \\
\left.\Delta h\right|_{y=0}=0 \quad x<\tilde{c} F(z),
\end{gathered}
$$




$$
\left.h\right|_{z=1}=\left.h\right|_{z=0} \mathrm{e}^{\mathrm{i} k_{3}},\left.\quad \frac{\partial h}{\partial z}\right|_{z=1}=\left.\frac{\partial h}{\partial z}\right|_{z=0} \mathrm{e}^{\mathrm{i} k_{3}},
$$

This system of equations, (5), is identical to that in Ayton \& $\mathrm{Kim}^{13}$ however now as we are considering a much broader range of leading edge geometries we must employ a different change of variables in order to solve it.

We choose the following variables similar to that by Roger et al. $(2013)^{17}$

$$
\begin{aligned}
\xi & =\frac{x}{\beta}-c F(z), \\
\eta & =y, \\
\zeta & =z,
\end{aligned}
$$

where $c=\tilde{c} / \beta$. This transformation converts the governing equation and boundary conditions, (5) to

$$
\begin{aligned}
\left(1+c^{2} F^{\prime}(\zeta)^{2}\right) \frac{\partial^{2} h}{\partial \xi^{2}}+\frac{\partial^{2} h}{\partial \eta^{2}}+\frac{\partial^{2} h}{\partial \zeta^{2}} & -2 c F^{\prime}(\zeta) \frac{\partial^{2} h}{\partial \xi \partial \zeta}-c F^{\prime \prime}(\zeta) \frac{\partial h}{\partial \xi}+(\delta M)^{2} h=0, \\
\left.\frac{\partial h}{\partial \eta}\right|_{\eta=0} & =-\mathrm{e}^{\mathrm{i} \delta \xi+\mathrm{i} k_{1} c F(\zeta)+\mathrm{i} k_{3} \zeta} \quad \xi>0 \\
\left.\Delta h\right|_{\eta=0} & =0 \quad \xi<0 \\
\left.h\right|_{\zeta=1} & =\left.h\right|_{\zeta=0} \mathrm{e}^{\mathrm{i} k_{3}} \\
\left.\frac{\partial h}{\partial z}\right|_{z=1} & =\left.\frac{\partial h}{\partial z}\right|_{z=0} \mathrm{e}^{\mathrm{i} k_{3}}
\end{aligned}
$$

where $\delta=k_{1} / \beta$, and derivatives of $F$ are taken in the weak sense at discontinuities.

This completes the formulation of the mathematical model.

\section{Analytic solution}

We proceed to solve (7) by following a similar set of steps as Ayton \& Kim,${ }^{13}$ inspired by Envia (1988), ${ }^{18}$ however take care over the new terms in the governing equations which were not present when $F(z)$ is restricted to a simple serration.

We first apply a Fourier transform in the $\xi$ variable,

$$
H(\lambda, \eta, \zeta)=\int_{-\infty}^{\infty} h(\xi, \eta, \zeta) \mathrm{e}^{\mathrm{i} \lambda \xi} d \xi
$$

then separate the solution into $\eta$ and $\zeta$ dependencies, $H(\lambda, \eta, \zeta)=Y(\lambda, \eta) Z(\lambda, \zeta)$, with separation constant $\chi$. This results in governing equations

$$
Y^{\prime \prime}+\left((\delta M)^{2}-\lambda^{2}-\chi^{2}\right) Y=0,
$$

and

$$
Z^{\prime \prime}+2 \mathrm{i} \lambda c F^{\prime} Z^{\prime}+\left(\mathrm{i} \lambda c F^{\prime \prime}-\lambda^{2} c^{2}\left(F^{\prime}\right)^{2}+\chi^{2}\right) Z=0
$$

Eq (9) has solutions

$$
Y(\lambda, \eta)=\operatorname{sgn}(\eta) \mathrm{e}^{-|\eta| \sqrt{\lambda^{2}-w^{2}}}
$$

where

$$
w^{2}=(\delta M)^{2}-\chi^{2}
$$

Eq (10) has solutions

$$
Z(\lambda, \zeta)=\mathrm{e}^{-\mathrm{i} \lambda c F(\zeta)}(A(\lambda) \cos (\chi \zeta)+B(\lambda) \sin (\chi \zeta)) .
$$

We impose the periodicity boundary conditions to $Z$ to solve for $\chi$ and eliminate one of $A, B$. This yields a spanwise orthogonal basis by

$$
Z_{n}(\lambda, \zeta)=\mathrm{e}^{-\mathrm{i} \lambda c F(\zeta)} \mathrm{e}^{\mathrm{i} k_{3} \zeta+2 n \pi \mathrm{i} \zeta},
$$


and a general solution given by

$$
H(\lambda, \eta, \zeta)=\sum_{n=-\infty}^{\infty} A_{n}(\lambda) \operatorname{sgn}(\eta) \mathrm{e}^{-|\eta| \sqrt{\lambda^{2}-w_{n}^{2}}} Z_{n}(\lambda, \zeta),
$$

where

$$
w_{n}^{2}=(\delta M)^{2}-\chi_{n}^{2}, \quad \chi_{n}= \pm k_{3}+2 n \pi .
$$

Now that we have the necessary orthogonal spanwise basis which is a generalisation of the basis used in Ayton \& $\mathrm{Kim}^{13}$ we proceed to solve for the $A_{n}$ following a similar Wiener-Hopf analysis to yield

$$
\tilde{A}_{n}^{+}(\lambda)=\frac{G_{n}^{+}(\lambda)}{\sqrt{\lambda+w_{n}}},
$$

with

$$
G_{n}^{+}(\lambda)=\frac{\mathrm{i}}{\lambda+\delta} \frac{1}{\sqrt{-\delta-w_{n}}}
$$

hence

$$
H(\lambda, \eta, \zeta)=\operatorname{sgn}(\eta) \sum_{n=-\infty}^{\infty} \frac{G_{n}^{+}(\lambda) E_{n}(\lambda) \mathrm{e}^{-|\eta| \sqrt{\lambda^{2}-w_{n}^{2}}}}{\sqrt{\lambda+w_{n}}} Z_{n}(\lambda, \zeta),
$$

where the $E_{n}$ are defined via

$$
\mathrm{e}^{\mathrm{i} k_{1} c F(\zeta)+\mathrm{i} k_{3} \zeta}=\sum_{n=-\infty}^{\infty} E_{n}(\lambda) Z_{n}(\lambda, \zeta) .
$$

The functions $E_{n}(\lambda)$ are known and dependent on the specific leading-edge geometry. For the serrated edge is is found that the $E_{n}$ are the key functions determining the overall magnitude of the far-field acoustic pressure and its level of modulation (i.e. how oscillatory it is in the far field). ${ }^{13}$

We invert the Fourier transform and obtain the far-field acoustics by applying the method of steepest descents to give

$$
\begin{aligned}
& h(r, \theta, z) \sim \\
& \sum_{n=-\infty}^{\infty} \frac{\mathrm{e}^{\pi \mathrm{i} / 4}}{\sqrt{\pi}} G_{n}^{+}\left(-w_{n} \cos \theta\right) E_{n}\left(-w_{n} \cos \theta\right) \cos \left(\frac{\theta}{2}\right) \frac{\mathrm{e}^{\mathrm{i} w_{n} r}}{\sqrt{r}} Z_{n}\left(-w_{n} \cos \theta, z\right) \mathrm{e}^{-\mathrm{i} w_{n} \cos \theta c F(\zeta)},
\end{aligned}
$$

where $(r, \theta, z)$ are cylindrical polar coordinates with origin corresponding to Cartesian origin $x=y=z=0$.

Acoustic pressure is determined from the modified potential via

$$
p=-\left(\frac{\partial h}{\partial x}-\frac{\mathrm{i} k_{1}}{\beta^{2}} h\right) \mathrm{e}^{-\mathrm{i} k_{1} M^{2} x / \beta^{2}} .
$$

This solution is identical to the solution presented in Ayton \& $\operatorname{Kim}^{13}$ when $F(z)$ is a sawtooth serration, however now is generalised for $F(z)$ defined by any piecewise linear function.

This completes our analytic solution of the scattered field.

\section{Experimental setup and instrumentation}

\section{A. Flat plates leading edge serrations}

For economy and ease of manufacture, a comparative study on noise reductions of different leading edge profiles was performed on flat plates situated within a turbulent flow. The flat plate with a mean chord (b) of $150 \mathrm{~mm}$ and span of $450 \mathrm{~mm}$ was constructed by joining together two $1 \mathrm{~mm}$ thick metallic sheets to allow serrated flat plate inserts $2 \mathrm{~mm}$ thick to be inserted between them. All corners were rounded and the trailing edge sharpened to eliminate vortex shedding noise. Further details of this flat plate construction can be found in. ${ }^{10}$

A total of 15 flat plate serrations, which includes 5 different serrations of varying $c$ of 1,2 and 4 were investigated to explore the sensitivity on noise reductions. For each case the serration wavelength, $\lambda$, is kept constant. Note that in this paper values of $\lambda$ are presented as quantities normalized on the mean chord $b$ although there is no evidence to suggest that this is a meaningful normalization parameter in determining noise reductions. 


\section{B. Open-jet test facility and instrumentation}

Far-field noise measurements were carried out at the Institute of Sound and Vibration Research's open-jet wind tunnel facility. The wind tunnel is located within the anechoic chamber, of dimension $8 \mathrm{~m} \times 8 \mathrm{~m} \times 8 \mathrm{~m}$ as shown in figure 2 . The walls are acoustically treated with glass wool wedges and the cut-off frequency is $80 \mathrm{~Hz}$. The nozzle has dimensions of $150 \mathrm{~mm}$ and $450 \mathrm{~mm}$ and provides a maximum flow speed of 100 $\mathrm{m} / \mathrm{s}$. A detailed description of the wind tunnel, including its characteristics, is presented by. ${ }^{19}$ To maintain two-dimensional flow around the flat plate, side plates are mounted to the nozzle exits which will also support the flat plate and aerofoil in the flow. The mean leading edge of the flat plate is located $150 \mathrm{~mm}$ downstream from the nozzle exit.

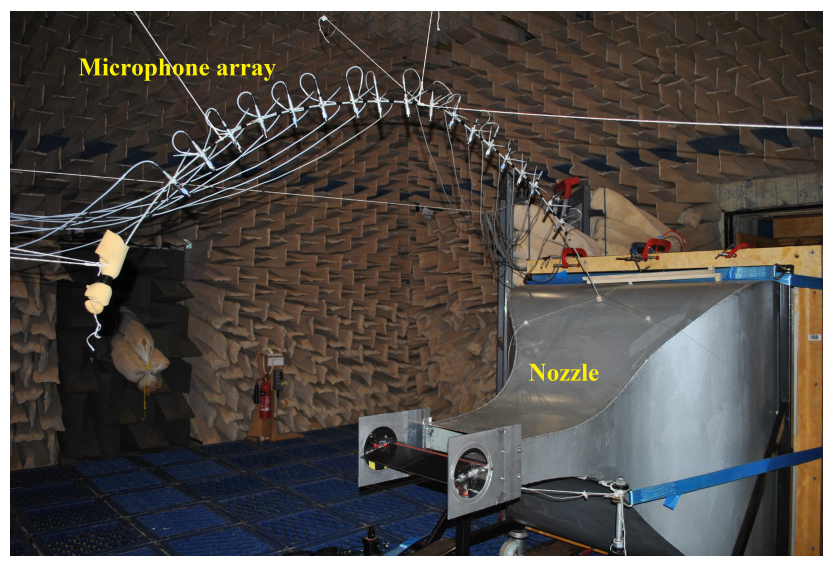

Figure 2: Photograph of jet nozzle and test setup inside the ISVR anechoic chamber.

In order to prevent tonal noise generation due to Tollmien-Schlichting waves convecting in the laminar boundary layer, and to ensure complete consistency between the different cases, the flow near the leading edge of the aerofoil was tripped to force transition to turbulence using a rough band of tape of width $1.25 \mathrm{~cm}$ located $16.6 \%$ of chord from the leading edge, on both suction and pressure sides. The tape has roughness of SS 100, corresponding to a surface roughness of $140 \mu \mathrm{m}$. Transition is forced by the use of the trip tape, which is many orders of magnitude rougher than the aerofoil surface, and is therefore highly unlikely to affect transition. Previous noise measurements in our facility have indicated that self-noise is insensitive to the method of tripping.

\section{Far-field noise measurements}

Far-field noise measurements from the flat plate were made using 11, half-inch condenser microphones (B\&K type 4189) located at a constant radial distance of $1.2 \mathrm{~m}$ from the mid span of the flat plate leading edge. These microphones are placed at emission angles of between $40^{\circ}$ and $140^{\circ}$ measured relative to the downstream jet axis. Measurements were carried for $10 \mathrm{~s}$ duration at a sampling frequency of $50 \mathrm{kHz}$, and the noise spectra was calculated with a window size of 1024 data points corresponding to a frequency resolution of $48.83 \mathrm{~Hz}$ and a $B T$ product of about 500, which is sufficient to ensure negligible variance in the spectral estimate at this frequency resolution.

The acoustic pressure at the microphone was recorded at the mean flow velocities $(U)$ of $20,40,60$ and $80 \mathrm{~m} / \mathrm{s}$. Noise reductions are presented in terms of the Sound Power Level spectra PWL $(f)$ calculated by integrating the pressure spectra over the polar array of 11 microphones using the procedure described in. ${ }^{10}$ Sound power level reductions are determined by subtracting the sound power level spectra due to the serrated flat plate from that due to the baseline straight edge profile.

\section{Turbulence characterisation}

A bi-planar rectangular grid with overall dimensions of $630 \times 690 \mathrm{~mm}^{2}$ located in the contraction section $75 \mathrm{~cm}$ upstream of the nozzle exit was used to generate turbulent flow that provides a velocity spectrum that 
is a close approximation to homogeneous and isotropic turbulence at the aerofoil leading edge. However, we emphasize that the condition of isotropy is not a key requirement for predicting the noise radiation but only that the velocity spectrum at the aerofoil leading edge is known. A comparison of the streamwise velocity spectra measured at $145 \mathrm{~mm}$ from the nozzle exit $\left(S_{u u} / U\right)$ plotted against $f / U$ is compared in figure 3 to the theoretical Liepmann velocity spectrum, where the mean square velocity and integral length scale are chosen to give best fit to the measured data. Close agreement is observed for $2.5 \%$ turbulence intensity and a $7.5 \mathrm{~mm}$ streamwise integral length-scale.

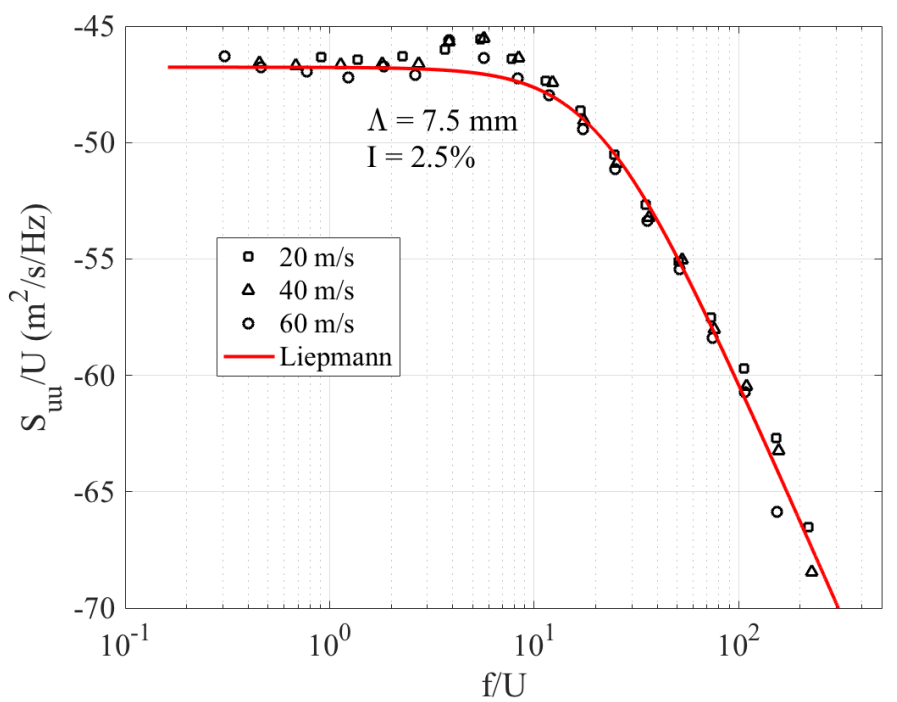

Figure 3: Comparison between the measured axial velocity spectra and theoretical Liepmann spectra.

\section{E. Effect of trailing-edge self noise}

The experimental setup cannot avoid the generation of trailing-edge self noise. This will become increasingly dominant at high frequencies $k_{1} \gtrsim 6$, as illustrated by Figure 4, moreso when the leading-edge geometry permits a significant reduction of leading-edge noise (therefore also affecting lower frequencies). As these frequencies are within the range we wish to consider for our experimental and analytical comparison, we must include a simple trailing-edge noise contribution to the analytical model.

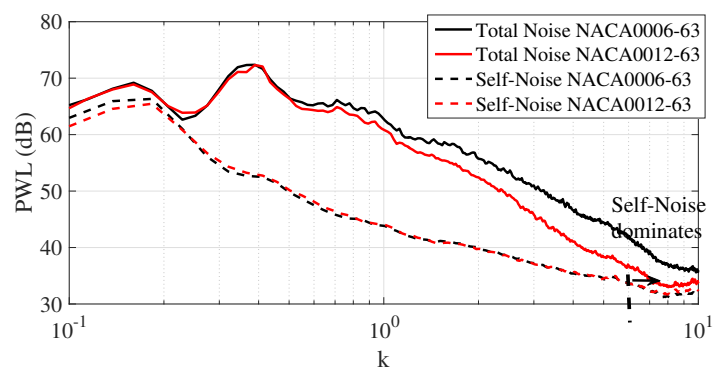

Figure 4: Far-field power spectra as a function of non-dimensionalised frequency $k=k_{1}$ for two different aerofoils. Self-noise measurements are obtained when the grid is not placed over the flow nozzle.

We use Amiet's trailing-edge noise model ${ }^{20}$ with Howe's approximation ${ }^{21}$ to Chase's wall-normal turbulent spectrum, ${ }^{22}$ as detailed in Glegg \& Devenport (2017) §9-10. ${ }^{23}$ This provides us with a far-field spectrum directly above the trailing edge, in the mid-span, of

$$
\mathrm{SPL}_{\mathrm{TE}}=10 \log _{10}\left(S_{p p}\left(0, r_{t}, 0, \omega_{t}\right)\right)
$$


where

$$
S_{p p}\left(x_{t}, y_{t}, 0, \omega_{t}\right) \approx \pi b\left(\frac{\omega_{t} c_{h} y_{t}}{4 \pi c_{\infty} r_{t}^{2}}\right)^{2} \phi_{p p}\left(\omega_{t}\right)|\mathcal{L}|^{2} .
$$

Here $\left(x_{t}, y_{t}, z_{t}\right)$ are standard Cartesian coordinates centred on the trailing edge, $r_{t}$ is the radial distance from the trailing edge, $b$ is the chord span, and $c_{h}$ is the chord length. The frequency, $\omega_{t}$, is assumed to satisfy $k_{1}=\omega_{t} / U_{c}$ where $U_{c}$ is the convection velocity within the boundary layer, $U_{c} \approx 0.7 U$. $\mathcal{L}$ is Amiet's generalised lift function [23, Eq (15.2.11)], and $\phi_{p p}$ is the expected wall pressure spectrum [23, Eq (9.2.37) denoted as $G_{p p}$ ]

\section{Results}

\section{A. Analytic directivity}

We first investigate the leading-edge far-field span-averaged directivity, defined as

$$
D_{a}(r, \theta)=\int_{0}^{1}|p(r, \theta, z)|^{2} d z
$$

for our five test-case leading-edge geometries.

In Figure 5 we plot $D_{a}(10, \theta)$ for $k_{3}=0, k_{1}=5$ and varying tip-to-root ratio $c$, for all five test cases. We consider a moderate $k_{1}$ value, as it is known that for low-frequency interactions $\left(k_{1} \lesssim 1\right)$ serrations are not very effective in reducing far-field noise. ${ }^{13}$ It is clear the square wave does not follow the same trend as the other four geometries, which consistently show a decrease of far-field noise as $c$ is increased. This implies one of the key mechanisms reducing noise as $c$ increases is not in effect for the square wave. It has been determined in Ayton \& $\mathrm{Kim}^{13}$ the two key mechanisms are a destructive interference, and a redistribution of energy to higher (non-propagating) modes. The interference is still present for the square wave, thus we expect it is the redistribution of modes which is less effective for the square wave than for the other leading-edge geometries.

Figure 5 also shows that different geometries are more effective at different values of $c$. For example, the v-root produces more noise than the u-root at $c=2$, but the u-root produces more noise than the v-root at $c=5$. Clearly the dual-mechanism of noise reduction (increased interference and redistribution of energy)

leads to complicated situations where different designs a optimal for different incident frequencies, since as frequency varies there is a shift in which mechanism is most prominent. Also clear is that there must be a strong dependency on the spanwise component of the incident turbulence given the edge has spanwise variation. Therefore to assess the noise reduction effects over a range of frequencies and to include a reliable spanwise dependency of the incident field we consider the far-field SPL and compare to experimental results.

\section{B. Comparison to Experimental Results}

The experimental results include a contribution from both leading-edge noise and trailing-edge noise, with the latter becoming increasingly important at higher frequencies and in situations where the leading-edge geometry significantly reduces the leading-edge contribution.

We therefore consider a combined leading-edge and trailing-edge SPL defined (for the analytic results) as

$$
\operatorname{SPL}\left(k_{1}\right)=\int_{-\infty}^{\infty}|p(r, \theta, z)|^{2} \Phi^{(\infty)}\left(k_{1}, k_{3}\right) d k_{3}+\mathrm{SPL}_{\mathrm{TE}}
$$

where $\Phi^{(\infty)}$ is an upstream turbulent spectra given by

$$
\Phi^{(\infty)}\left(k_{1}, k_{3}\right)=\frac{L^{2}\left(k_{1}^{2}+k_{3}^{2}\right)}{\left(1+L^{2}\left(k_{1}^{2}+k_{3}^{2}\right)\right)^{5 / 2}},
$$

with $L$ the integral lengthscale of turbulence.

In each experimental case the wavelength of the leading-edge geometry is $\lambda^{*}=25 \mathrm{~mm}$ and the total chord length is $b^{*}=150 \mathrm{~mm}$. The flow speed is fixed at $u^{*}=60 \mathrm{~ms}^{-1}$. The tip-to-root ratios corresponding to $c=1,2,4$ are considered. At the lowest frequencies $f^{*}<1,000 \mathrm{~Hz}$ it is expected the experimental results contain significant amounts of unavoidable jet noise. 


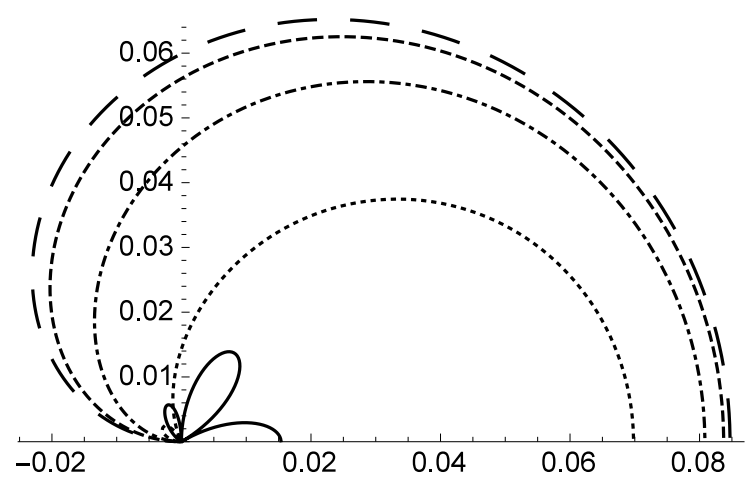

(a) Serration

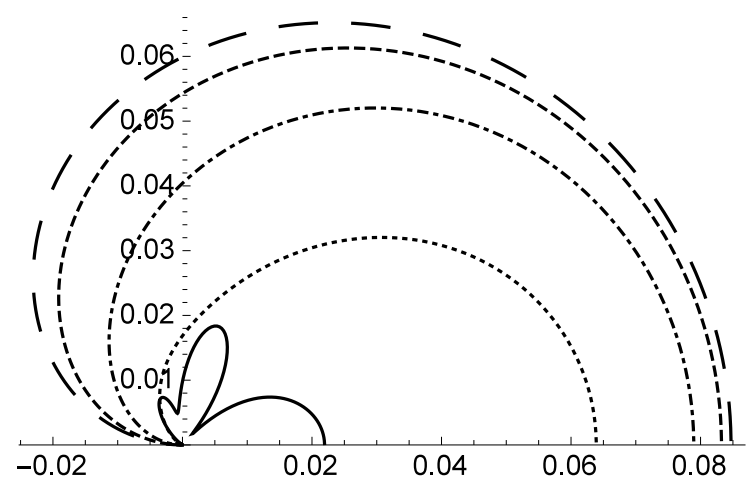

(c) U-root

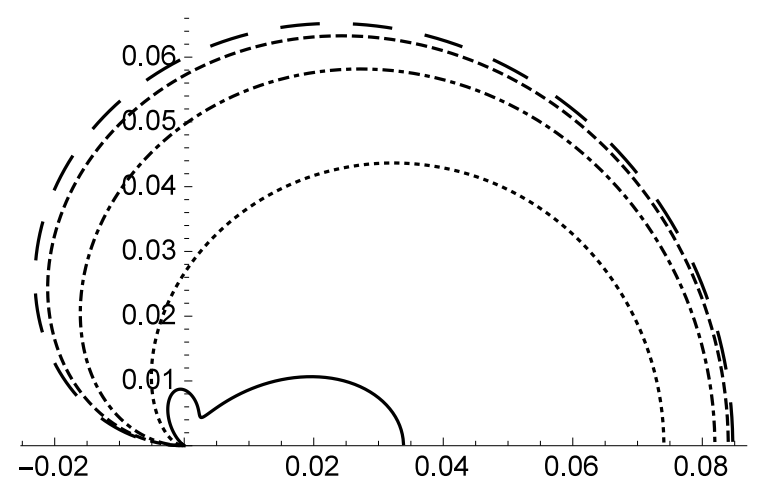

(b) V-root

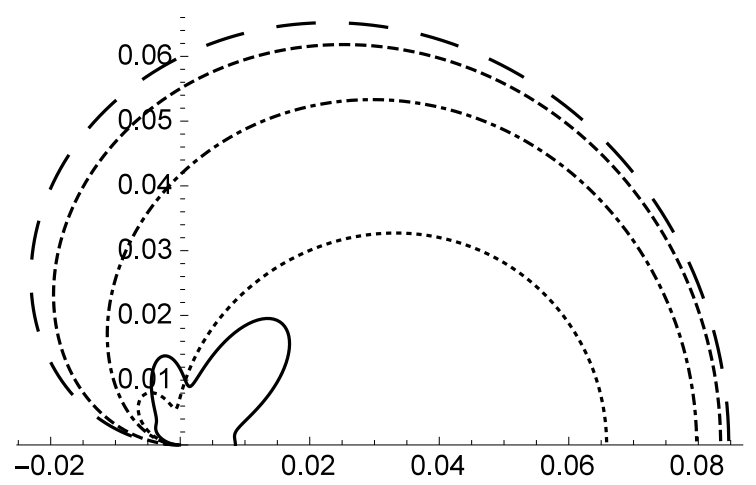

(d) Chopped peak

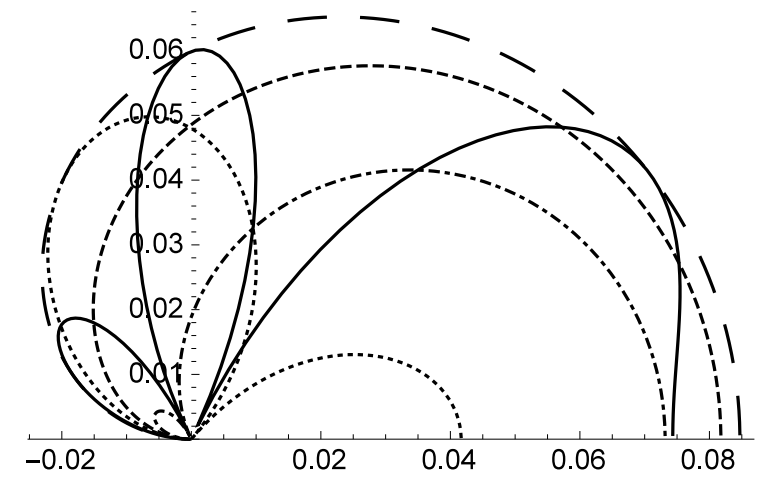

(e) Square wave

Figure 5: Average directivity over the channel span, $D_{a}(r, \theta)$ as given by $(25)$, for $r=10, M=0.5, k_{3}=0$. Large dashed $c=0$; dashed $c=0.5$; dot-dashed $c=1$; dotted $c=2$, solid $c=5$. 
In Figures 6, 7, and 8 we plot the analytic results against the experimental measurements for $c=$ 1,2, 4 respectively, and for each leading edge geometry. The straight edge result is identical in all figures and provides a reference point for considering the noise reduction. The dashed lines are the experimental measurements whilst the solid lines are the analytic results. Overall we see good agreement between the analytic predictions and the experimental measurements for all edges therefore this mathematical model is accurately capturing the key physics of the fluid-structure interaction.

The comparison is particularly good for the sawtooth (a), chopped peak (d), and square wave (e) geometries over all tip-to-root ratios. The very small slitted root regions in the v-root (b) and u-root (c) could allow non-linear or viscous effects to be more prominent in the experimental results than for the other geometries (where there are no narrow regions); these effects are not accounted for in the mathematical model.

\section{Isolated leading-edge noise}

The results in the previous subsection include the effects of (straight) trailing-edge noise as this is unavoidable during experimental testing. The analytic results however allow us to isolate just the leading-edge noise. We wish to do this because simultaneous to the development of quieter leading-edge designs, is the development of quieter trailing-edge designs. ${ }^{24-28}$ If the trailing-edge noise is significantly reduced from its straight-edge level at a given (high) frequency, understanding how a leading-edge adaptation affects the leading-edge noise at that frequency becomes important.

We use the analytic results to plot the SPL due only to the leading edge in Figure 9 compared to the SPL when trailing-edge noise is included. We calculate the isolated leading-edge SPL from (26) by neglecting the contribution from $\mathrm{SPL}_{\mathrm{TE}}$. We see the key difference as expected is at the high-frequency end where trailing-edge noise is most dominant. Without the inclusion of trailing-edge noise, the leading-edge designs continue to increase noise reduction as frequency increases, except the square wave (e) which is ineffective at high-frequencies. When trailing-edge noise is included each leading-edge geometry performs identically at high frequencies, however when trailing-edge noise is neglected we see clearly the sawtooth out-performs the other geometries at high frequencies, despite being less effective than the other geometries at mid-range frequencies (e.g. at $k_{1} \sim 2.5$ the square wave (e) is much better at reducing noise than the other geometries).

\section{Conclusions}

This paper has presented an analytic solution for the leading-edge noise generated by a semi-infinite flat plate with arbitrary piecewise-linear leading-edge geometry. For comparison with experimental results, a simple model of trailing-edge noise ${ }^{20}$ is added to the leading-edge noise results. The trailing-edge noise model does not account for the backscattering of the acoustic field ${ }^{29}$ by the leading edge, similarly the leading-edge noise model does not account for any rescattering by the straight trailing edge. These smaller effects would be expected to introduce oscillations into the SPL where interference between rescattered fields could occur. Overall there is good agreement between the analytical results and experimental measurements for all geometries at a variety of tip-to-root ratios.

Key to this model is the separation of spanwise and normal variables after applying a Fourier transform in the streamwise direction. Because of this, the new mathematical formulation is simpler to implement than the previous (spanwise) Fourier decomposition model of Lyu et $\mathrm{al}^{15}$ which involves an iterative numerical procedure to obtain far-field results.

The analytic results have been used to isolate just the leading-edge noise at high-frequencies, which can't be done easily experimentally. Different behaviour has been identified between the different test case geometries at high-frequencies when only the leading-edge noise is considered. In particular, the sawtooth is most effective at high frequencies despite not being the most effective at mid-range frequencies.

These results provide groundwork for optimising leading-edge geometries for reducing aerofoil-turbulence interaction noise.

\section{References}

\footnotetext{
${ }^{1}$ Olsen, W., \& Wagner, J. 1982 Effect of Thickness on Airfoil Surface Noise. AIAA Journal 20, 437-439.

${ }^{2}$ Ayton, L. J. 2016 An analytic solution for gust-aerofoil interaction noise including effects of geometry. IMA Journal of Applied Mathematics 82 280-304.
} 


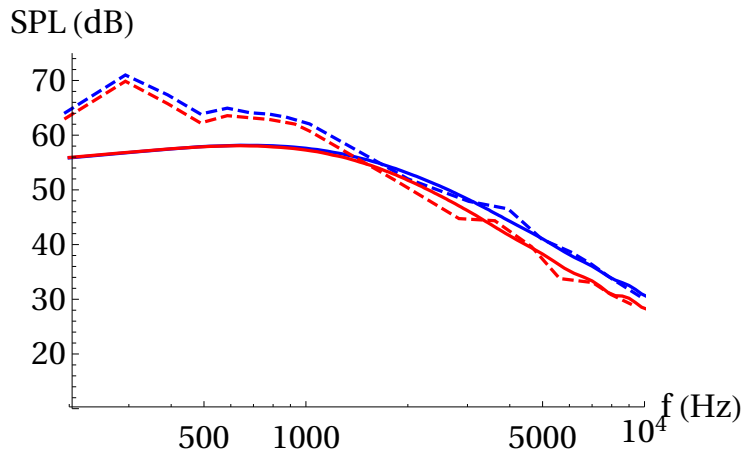

(a) sawtooth

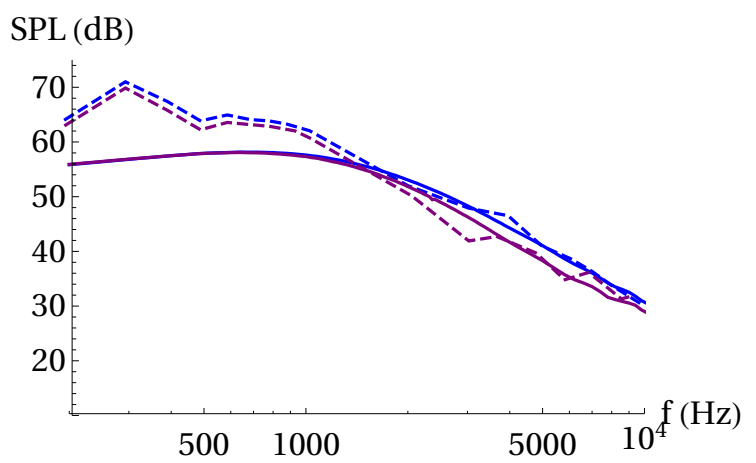

(c) u-root
SPL (dB)

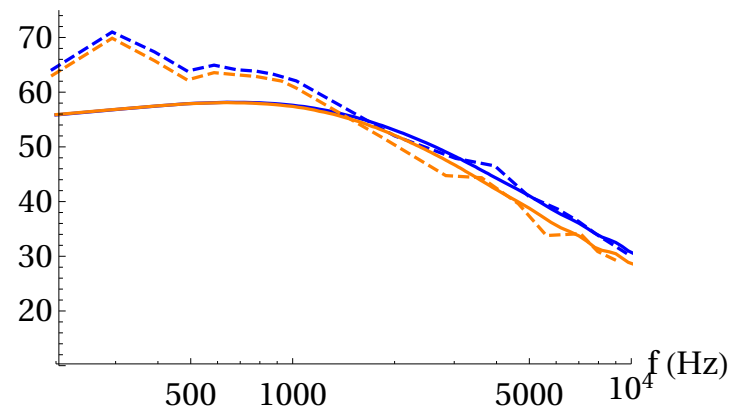

(b) v-root

SPL (dB)

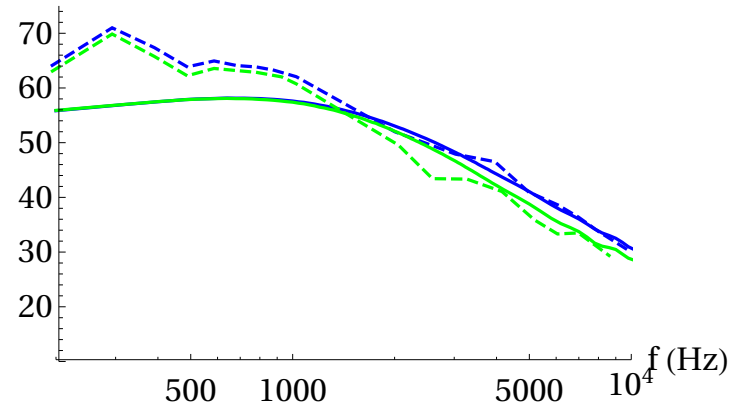

(d) chopped peak

$\operatorname{SPL}(\mathrm{dB})$

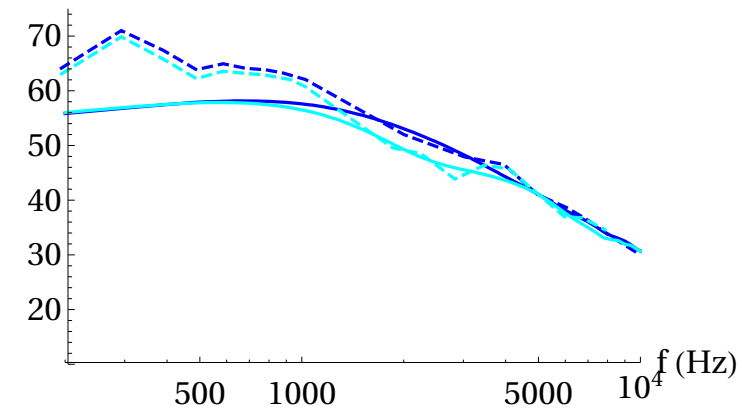

(e) square wave

Figure 6: SPL from analytic results (solid) and experimental measurements (dashed) for each of the five test case geometries: red (a), orange (b), purple (c), green (d), cyan (e). The straight-edge results are blue throughout. In each case $M=0.17, c=1$. 


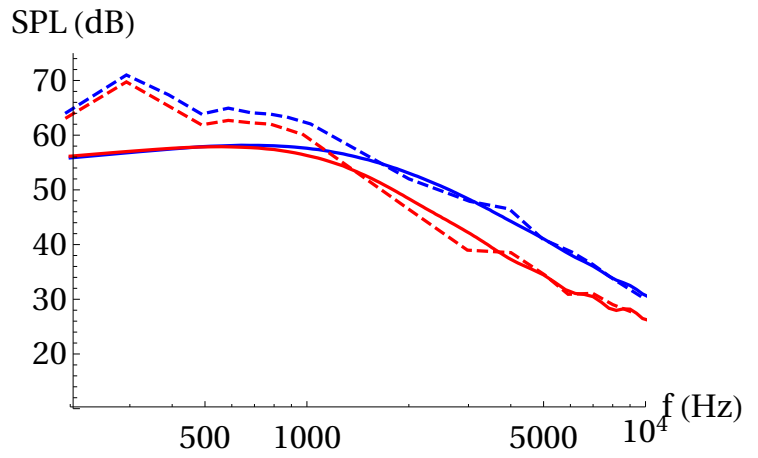

(a) sawtooth

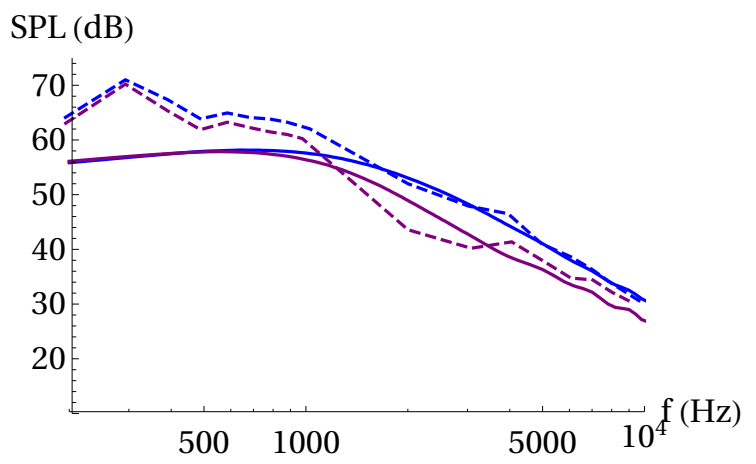

(c) u-root
SPL (dB)

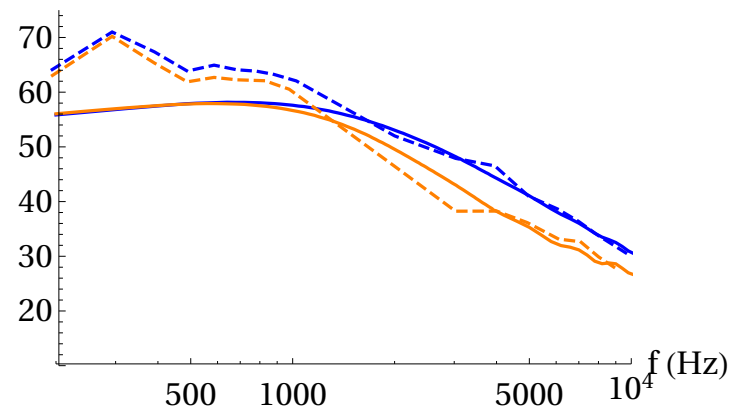

(b) v-root

SPL (dB)

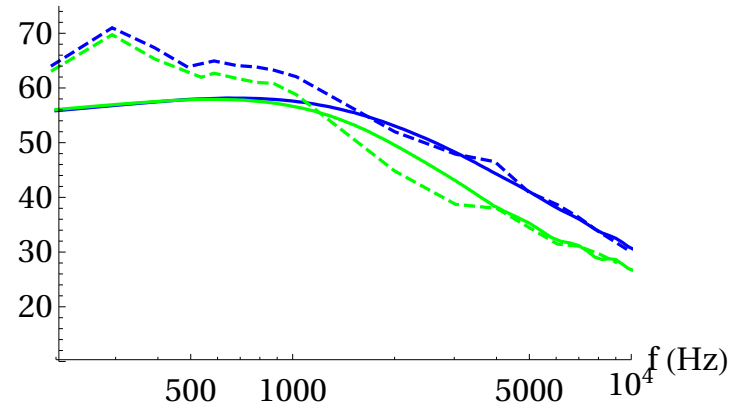

(d) chopped peak

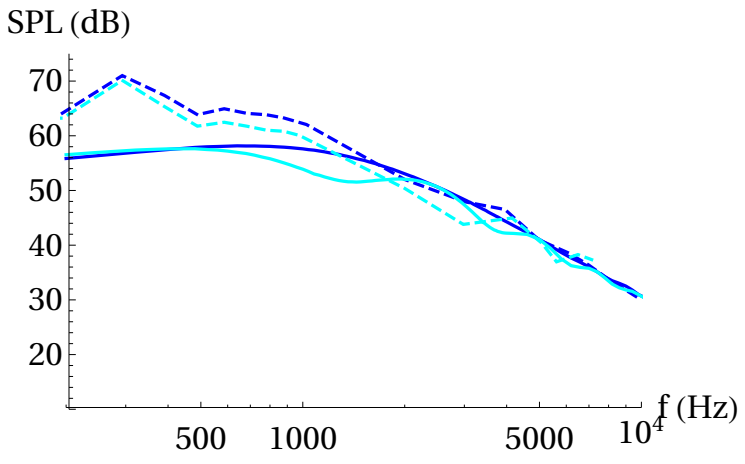

(e) square wave

Figure 7: SPL from analytic results (solid) and experimental measurements (dashed) for each of the five test case geometries: red (a), orange (b), purple (c), green (d), cyan (e). The straight-edge results are blue throughout. In each case $M=0.17, c=2$. 


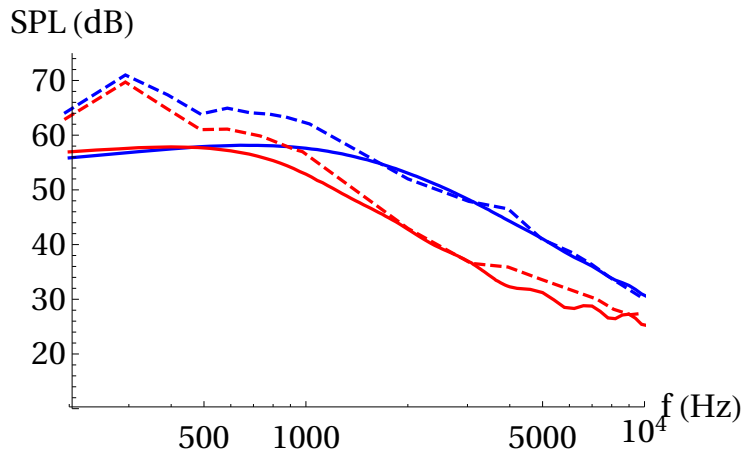

(a) sawtooth

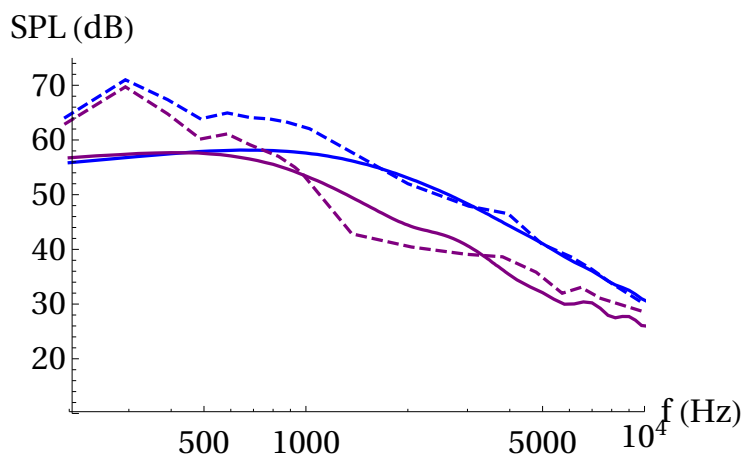

(c) u-root
SPL $(\mathrm{dB})$

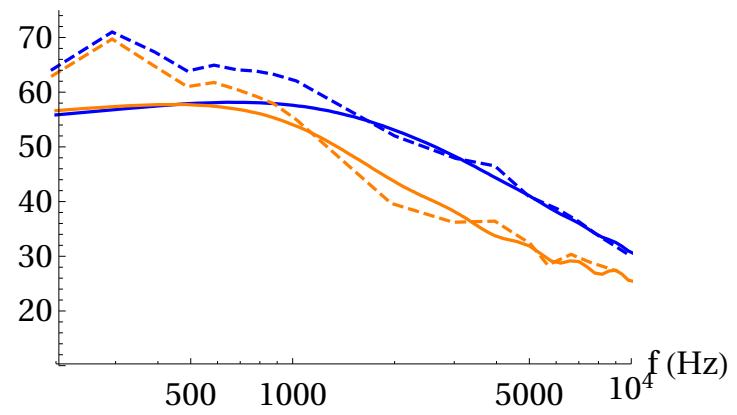

(b) v-root

SPL (dB)

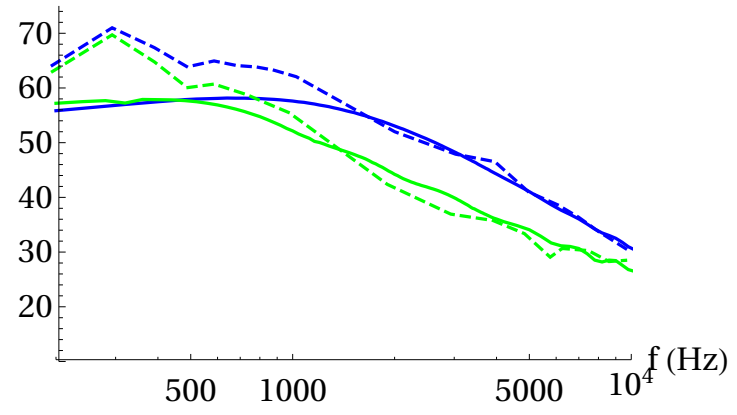

(d) chopped peak

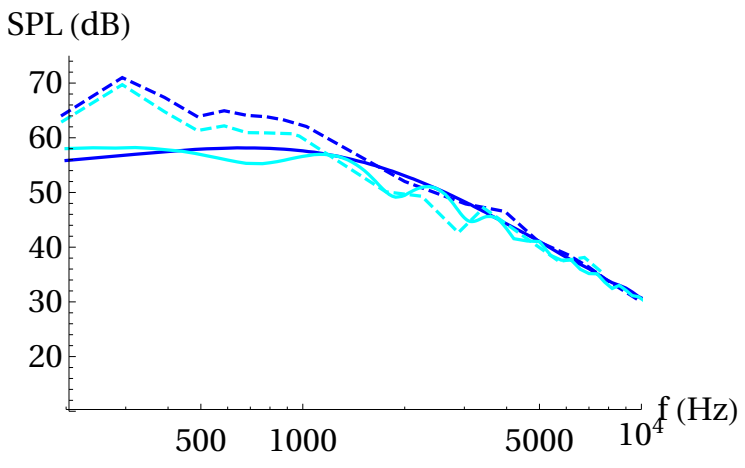

(e) square wave

Figure 8: SPL from analytic results (solid) and experimental measurements (dashed) for each of the five test case geometries: red (a), orange (b), purple (c), green (d), cyan (e). The straight-edge results are blue throughout. In each case $M=0.17, c=4$. 


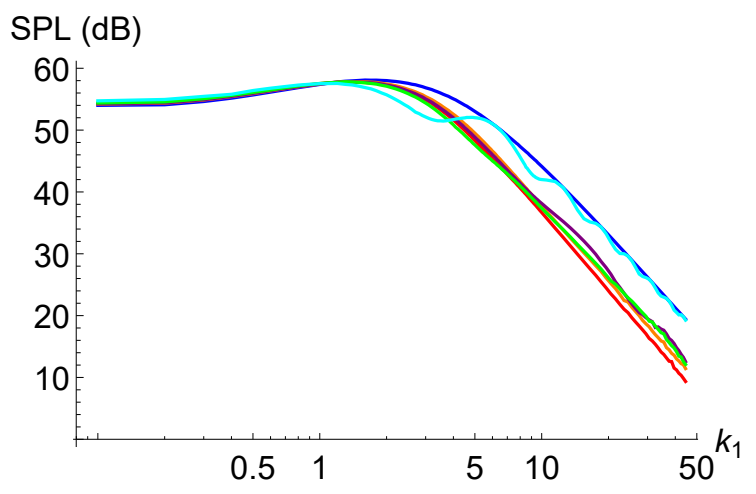

(a) leading edge contribution only

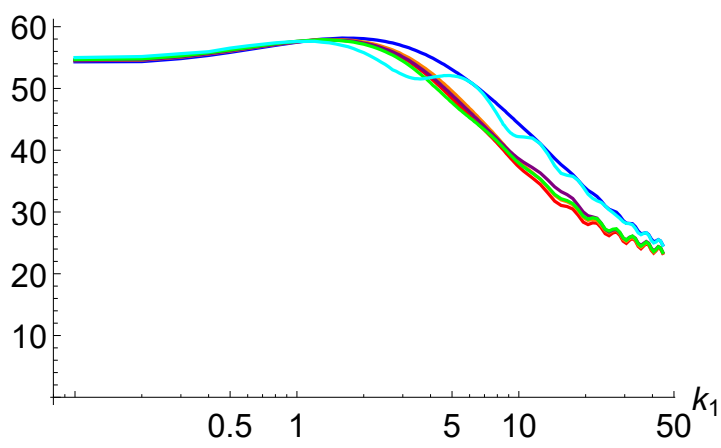

(b) including trailing-edge noise

Figure 9: SPL from analytic results for each of the five test case geometries: red (a), orange (b), purple (c), green (d), cyan (e). The straight-edge result in blue is provided for comparison. In each case $M=0.17$, $c=2$.

${ }^{3}$ Ayton, L. J. \& Chaitanya, P. 2017 Analytical and experimental investigation into the effects of leading-edge radius on gust-aerofoil interaction noise Journal of Fluid Mechanics $\mathbf{8 2 9} 780-808$.

${ }^{4}$ Graham, R. R. 1934 The silent flight of owls. Journal of the Royal Aeronautical Society 38, 837-843.

${ }^{5}$ Lilley G. M. 1998 A study of the silent flight of the owl. 4th AIA A/CEAS Aeroacoustics Conference, Toulouse, AIAA 1998-2340.

${ }^{6}$ Kim, J.-W., Haeri, S. \& Joseph, P. F. 2016 On the reduction of aerofoil-turbulence interaction noise associated with wavy leading edges. Journal of Fluid Mechanics 792, 526-552.

${ }^{7}$ Lau, A. S. H., Haeri, S. \& Kim, J. W. 2013 The effect of wavy leading edges on aerofoil-gust interaction noise. Journal of Sound and Vibration 332, 6234-6253.

${ }^{8}$ Clair, V., Polacsek, C., Le Garrec, T., Reboul, G., Gruber, M. \& Joseph, P. 2013 Experimental and numerical investigation of turbulence-airfoil noise reduction using wavy edges AIAA Journal 51 2695-2713.

${ }^{9}$ Chaitanya, P., Narayanan, S., Joseph, P. F. \& Kim, J.-W. 2016 Leading edge serration geometries for significantly enhanced leading edge noise reductions. 22nd AIAA/CEAS Aeroacoustics 2736.

${ }^{10}$ S. Narayanan and P. Chaitanya and S. Haeri and P. Joseph and J. W. Kim and C. Polacsek 2015 Airfoil noise reductions through leading edge serrations. Physics of Fluids $\mathbf{2 7}$.

${ }^{11}$ Hansen, K., Kelso, R. \& Doolan, C. 2012 Reduction of flow induced airfoil tonal noise using leading edge sinusoidal modifications. Acoustics Australia 40 172-177.

${ }^{12}$ Biedermann, T. M., Chong, T. P., Kameier, F. \& Paschereit, C. O. 2017 Statistical empirical modeling of airfoil noise subjected to leading-edge serrations. AIAA Journal $553128-3142$.

${ }^{13}$ Ayton, L. J. \& Kim, J.-W. 2017 An analytic solution for the noise generated by gust-aerofoil interaction for plates with serrated leading edges. Journal of Fluid Mechanics submitted.

${ }^{14}$ Huang, X. 2017 Theoretical model of acoustic scattering from a flat plate with serrations. Journal of Fluid Mechanics 819, $228-257$.

${ }^{15}$ Lyu, B., \& Azarpeyvand, M. 2017 On the noise prediction for serrated leading edges . ArXiv 1706.04509.

${ }^{16}$ Mathews, J. \& Peake, N. 2015 Noise generation by turbulence interacting with an aerofoil with a serrated leading edge. 21st AIAA/CEAS Aeroacoustics Conference, Dallas, TX .

${ }^{17}$ Roger, M., Schram, C. \& De Santana, L. 2013 Reduction of airfoil turbulence-impingement noise by means of leading-edge serrations and/or porous material, 19th AIAA/CEAS Aeroacoustics, AIAA 2013-2108.

${ }^{18}$ Envia, E. 1988 Influence of Vane Sweep on Rotor-Stator Interaction Noise.. PhD thesis, University of Arizona.

${ }^{19}$ Chong, T. P., Joseph, P. F. \& Davis, P. O. A. L. 2008 A parametric study of passive flow control for a short, high area ratio $90^{\circ}$ curved diffuser. Journal of Fluids Engineering 130, 111104-12.

${ }^{20}$ Aмiet, R.K. 1976 Noise due to turbulent flow past a trailing edge. Journal of Sound and Vibration 47 $387-393$.

${ }^{21}$ Howe, M. S. 1998 Acoustics of Fluid-structure Interactions Cambridge University Press.

${ }^{22}$ ChASE, D. M. 1987 The character of the turbulent wall pressure spectrum at subconvective wavenumbers and a suggested comprehensive model. Journal of Sound and Vibration 112, 125-147.

${ }^{23}$ GlegG, S. \& Devenport, W. 2017 Aeroacoustics of Low Mach Number Flows Academic Press, Elsevier.

${ }^{24}$ Van der Velden, W. C., Avallone, F. \& Ragni, D. 2017 Numerical analysis of noise reduction mechanisms of serrated trailing edges under zero lift condition. 23rd AIAA/CEAS Aeroacoustics Conference, AIAA, 2017-4173.

${ }^{25}$ Wolf, A., Lutz, T., Würz, W., Krämer, E., Stalnov, O., \& Seifert, A. 2014 Trailing edge noise reduction of wind turbine blades by active flow control. Wind Energy 18, 909-923.

${ }^{26}$ JaWorski, J.W. \& Peake, N. 2013 Aerodynamic noise from a poroelastic edge with implications for the silent flight of owls. Journal of Fluid Mechanics 723, 456-479.

${ }^{27}$ Herr, M. 2006 Experimental study on noise reduction through trailing-edge brushes. Notes on Numerical Fluid Mechanics 92, 365-372. 
${ }^{28}$ Clark, I.A, Daly, C. A., Devenport, W., Alexander, W. N., Peake, N., Jaworski, J.W., \& Glegg, S. 2016 Bio-inspired canopies for the reduction of roughness noise. Journal of Sound and Vibration, $38533-54$.

${ }^{29}$ Roger, M. \& Moreau, S. 2005 Back-scattering correction and further extensions of Amiet's trailing-edge noise model. Journal of Sound and Vibration 286, 477-506. 